PROCEEDINGS OF THE

AMERICAN MATHEMATICAL SOCIETY

Volume 136, Number 6, June 2008, Pages 1919-1923

S 0002-9939(08)09250-2

Article electronically published on February 7, 2008

\title{
FREE SUBMODULES FOR THE CENTRAL REPRESENTATION IN THE COHOMOLOGY OF LIE ALGEBRAS
}

\author{
GRANT CAIRNS AND BARRY JESSUP \\ (Communicated by Paul Goerss)
}

\begin{abstract}
If $Z$ is the centre of the Lie algebra $L$, its cohomology $H^{*}(L)$ is naturally a module over the exterior algebra $\Lambda Z$. Under suitable hypotheses on $L$, motivated by recent work by Pouseele and Tirao, we find free summands in $H^{*}(L)$ for this module structure, thus establishing the Toral Rank Conjecture for a new class of Lie algebras.
\end{abstract}

\section{INTRODUCTION}

We consider finite-dimensional complex Lie algebras $L$. Recall that for such an algebra $L$, the Toral Rank Conjecture (TRC) [4] states that

$$
\operatorname{dim} H^{*}(L) \geq 2^{\operatorname{dim} Z},
$$

where $Z$ is the centre of $L$, and $H^{*}(L)$ denotes the cohomology with trivial coefficients. The TRC is known to hold for nilpotent Lie algebras of dimension at most 14 [1. It holds for two-step nilpotent Lie algebras (see [6] and 1]) and more generally for positively graded Lie algebras where the centre is the summand of highest grading (see [3] and [7]). Recently Hannes Pouseele and Paulo Tirao gave a remarkably simple result, which establishes the TRC for a class of Lie algebras that includes algebras of large nilpotency class that are not positively graded [5]. The aim of this paper is to show that the argument in [5] can be extended to a larger class of algebras and that the conclusion of their theorem can also be strengthened.

The key idea is to note that the cohomology $H^{*}(L)$ is naturally a module over the exterior algebra $\Lambda Z$ and to look for free summands in $H^{*}(L)$ :

Theorem. Suppose that the Lie algebra $L$ is a direct sum of non-trivial subalgebras $A, B, C$, where $C$ is central and $A, B$ and $L$ are unimodular. Then the cohomology $H^{*}(L)$, as a $\Lambda C$-module, contains a free module on two generators.

This extends [5, Theorem 1], which is the case where it is assumed that $A$ and $B$ are abelian and that $B \oplus C$ is an ideal of $L$. Note that the unimodularity hypothesis on $A, B$ and $L$ is satisfied when $L$ is nilpotent, for example.

Received by the editors October 25, 2006.

2000 Mathematics Subject Classification. Primary 17B55, 17B56; Secondary 55 P62.

This research was supported in part by NSERC and the ARC.

The second author would like to thank the members of the Department of Mathematics and Statistics at La Trobe University for their hospitality during his stay there.

(C)2008 American Mathematical Society Reverts to public domain 28 years from publication 
Corollary. The cohomology $H^{*}(L)$ has dimension at least $2^{\operatorname{dim}(C)+1}$.

This strengthens [5, Corollary 2], which gives $\operatorname{dim} H^{*}(L) \geq 2^{\operatorname{dim}(C)}$. We give examples later to show that $C$ may indeed be the centre $Z$ of $L$. We also use the theorem to indicate how one may, for example, analyse the $\Lambda Z$ structure of the cohomology of the free two-step algebras on $m$ generators, where (except for $m=2$ ), there are no free submodules.

\section{Preliminaries}

If $L$ is a Lie algebra, and $L^{*}$ denotes the dual, its cohomology is obtained as follows: let $d: L^{*} \rightarrow \Lambda^{2} L^{*}$ be the transpose of the bracket [, ] $: \Lambda^{2} L \rightarrow L$, and extend it to a derivation of the Koszul complex $\Lambda L^{*}$ of degree 1 . The Jacobi identity is equivalent to $d^{2}=0$, and the cohomology $H^{*}(L)=H^{*}\left(\Lambda L^{*}, d\right)$ is the graded algebra defined as $H^{*}(L)=\operatorname{ker} d / \operatorname{im} d$.

If $x \in L, i_{x}$ denotes the derivation of $\Lambda L^{*}$ extending the natural map $x \in\left(L^{*}\right)^{*}$ to a derivation of $\Lambda L^{*}$ of degree -1 , and, when extended using the cap product $\Lambda Z \otimes \Lambda L^{*} \rightarrow \Lambda L^{*}$, makes $\Lambda L^{*}$ a module over $\Lambda Z$. The Lie derivative $\mathcal{L}_{x}=i_{x} d+d i_{x}$ is the extension of the transpose of $a d(x): L \rightarrow L$ to a derivation of degree 0 of $\Lambda L^{*}$, and $x$ belongs to the centre $Z(L) \Longleftrightarrow \mathcal{L}_{x}=0$. Thus if $x \in Z(L), i_{x}$ induces a derivation of the algebra $H^{*}(L)$, and the $\Lambda Z$-module structure on $\Lambda L^{*}$ induces one on $H^{*}(L)$. In 2, the homomorphism $\Lambda Z \rightarrow \operatorname{End}\left(H^{*}(L)\right)$ defining this module structure is called the central representation.

A Lie algebra is unimodular if trace $a d(x)=0$ for all $x \in L$, and it is easy to show that this is equivalent to $d: \Lambda^{\operatorname{dim} L-1} L^{*} \rightarrow \Lambda^{\operatorname{dim} L} L^{*}$ being zero. For $L$ as in the statement of the theorem, we choose bases for $A, B$ and $C$, and relative to the resulting basis for $L$, we define the Hodge star $\star: \Lambda^{k} L^{*} \rightarrow \Lambda^{\operatorname{dim} L-k} L^{*}$ in the usual manner. It is straightforward to show that if $L$ is unimodular, $H^{*}(L)$ is isomorphic to the space of harmonic forms (see [2], for example); recall that a form $\alpha$ is harmonic if $d \alpha=0$ and $d \star \alpha=0$.

The approach used in 2] (and independently in 5]) can be interpreted as follows ([5] uses homology whereas we will use cohomology in this paper): suppose there exists a closed $p$-form $\alpha \in \Lambda L^{*}$ such that the submodule $\Lambda Z \cdot[\alpha]$ of $H^{*}(L)$ generated by $\alpha$ is free. This occurs if and only if the $(p-k)$-form $i_{z_{1}} i_{z_{2}} \ldots i_{z_{k}} \alpha$ is not exact, where $\left\{z_{1}, \ldots, z_{k}\right\}$ is any basis for the centre $Z$. Then, if $\left\{z_{j_{1}}, z_{j_{2}}, \ldots, z_{j_{l}}\right\}$ is any subset of $\left\{z_{1}, \ldots, z_{k}\right\}$, the classes $\left[i_{z_{j_{1}}} i_{z_{j_{2}}} \ldots i_{z_{j_{l}}} \alpha\right]$ are all linearly independent, and hence $\operatorname{dim} H^{*}(L) \geq 2^{\operatorname{dim} Z}$. In this case, the central representation is faithful.

The result in $[5]$ is obtained by taking suitable hypotheses on an ideal $I$ of $L$ so that such an $\alpha$ is given by the pullback to $L$ of a nonzero form in $\Lambda^{\operatorname{dim} L / I} L / I$. (As noted in [2, there are many examples of Lie algebras where the central representation is not faithful; 2 shows that $H^{*}(L)$ is actually a module over a much larger algebra containing $\Lambda Z$ and begins the study of that module structure with the TRC as a goal.) The result [5] and our theorem above give examples where the central representation is faithful.

\section{Proof of the THEOREM}

Proof. Let $L^{*}$ denote the dual of $L$, and define subspaces $U=(B \oplus C)^{\perp}, V=$ $(A \oplus C)^{\perp}$ and $W=(A \oplus B)^{\perp}$ of $L^{*}$. The Koszul complex of $L$ can then be written 
as $(\Lambda U \otimes \Lambda V \otimes \Lambda W ; d)$. The fact that $A$ and $B$ are subalgebras and that $C$ is central implies that

$$
\begin{aligned}
& d: U \rightarrow \Lambda^{2} U \oplus(U \otimes V), \\
& d: V \rightarrow \Lambda^{2} V \oplus(U \otimes V), \text { and } \\
& d: W \rightarrow U \otimes V .
\end{aligned}
$$

Now let $\sigma, \varepsilon, \tau$ be nonzero elements in $\Lambda^{\operatorname{dim} U} U, \Lambda^{\operatorname{dim} V} V$ and $\Lambda^{\operatorname{dim} W} W$ respectively. Thus $\sigma \varepsilon \tau$ is a nonzero element in $\Lambda^{\operatorname{dim} L} L$.

We shall show that the unimodularity assumptions imply that $d \sigma=0$, and that the class $[\sigma] \in H^{\operatorname{dim} U}(L)$ is nonzero. If $\left\{z_{1}, \ldots, z_{k}\right\}$ is a basis for $C$ and $z=z_{1} \ldots z_{k} \in \Lambda^{k} C$, we will then have $\left[i_{z} \sigma \tau\right]= \pm[\sigma] \neq 0$, and so the $\Lambda C$ module generated by $[\sigma \tau]$ is free. An identical argument will show that the $\Lambda C$ module generated by $[\tau \varepsilon]$ is also free.

To show that $d \sigma=0$, first note that the unimodularity of the Lie algebra $L$ is equivalent to the condition

$$
0=\mathcal{L}_{x}(\sigma \varepsilon \tau), \quad \forall x \in L
$$

As $C$ is central, $\mathcal{L}_{x} \tau=0$ and so

$$
0=\left(\mathcal{L}_{x} \sigma\right) \tau \varepsilon+\sigma\left(\mathcal{L}_{x} \tau\right) \varepsilon, \quad \forall x \in L
$$

Now let $x \in B$, write $d_{\left.\right|_{V}}=\bar{d}_{V}+\theta$, with $\bar{d}_{V}: V \rightarrow \Lambda^{2} V$ and $\theta: V \rightarrow U \otimes V$, and denote $\overline{\mathcal{L}}_{x}=i_{x} \bar{d}_{V}+\bar{d}_{V} i_{x}$. The unimodularity of $B$ then gives $\sigma \varepsilon\left(\mathcal{L}_{x} \tau\right)=$ $\sigma \varepsilon\left(\overline{\mathcal{L}}_{x} \tau\right)=0$ as well. Hence, for $x \in B$, we have

$$
0=\left(\mathcal{L}_{x} \sigma\right) \tau \varepsilon=\left(\left(i_{x} d+d i_{x}\right) \sigma\right) \tau \varepsilon=\left(i_{x} d \sigma\right) \tau \varepsilon .
$$

Since $\sigma \in \Lambda^{\operatorname{dim} U} U$, (1) implies $d \sigma=\sigma \otimes v$ for some $v \in V$. Thus $0=\left(i_{x} d \sigma\right) \tau \varepsilon$ gives $0=\left(i_{x} d \sigma\right)$, and so $v=0$. Hence, $d \sigma=0$.

Hence by $(3), d(\sigma \tau)=(d \sigma) \tau=0$. Now, since the hypotheses are symmetric in $U$ and $V$, a similar argument shows that $d \varepsilon=0$ and $d(\varepsilon \tau)=0$. We also know that $\sigma= \pm \star \varepsilon \tau$, where $\star$ denotes the Hodge star map, so $\sigma$ is harmonic and thus $[\sigma] \neq 0$. By the same reasoning, $[\varepsilon] \neq 0$. Hence, the $\Lambda C$ modules generated by $[\sigma \tau]$ and $[\varepsilon \tau]$ are free.

\section{EXAmple}

Let $\mathfrak{f}_{n}$ denote the $(m+1)$-dimensional standard filiform algebra with basis $\left\{x_{0}\right.$, $\left.\ldots, x_{n}\right\}$ and relations $\left[x_{0}, x_{i-1}\right]=x_{i}, 2 \leq i \leq n$, and let $\mathfrak{h}_{m}$ denote the $(2 m+1)$ dimensional Heisenberg algebra with basis $\left\{y_{1}, \ldots, y_{m}, z_{1}, \ldots, z_{m}, w\right\}$ and relations $\left[y_{i}, z_{i}\right]=w, 1 \leq i \leq m$. Consider the extension of $\mathfrak{f}_{n} \oplus \mathfrak{h}_{m}$ defined by introducing the symbols

$$
a_{1}, \ldots, a_{m}, b_{1}, \ldots, b_{n-1}, c_{0}, \ldots, c_{m}
$$


and defining relations as follows:

$$
\begin{aligned}
{\left[x_{0}, z_{i}\right] } & =a_{i}, \quad 1 \leq i \leq m, \\
{\left[x_{0}, y_{1}\right] } & =-b_{1}, \\
{\left[x_{0}, b_{i-1}\right] } & =-b_{i}, \quad 2 \leq i \leq n-1, \\
{\left[x_{n}, y_{1}\right]=\left[x_{j}, b_{n-j}\right] } & =c_{0}, \quad 1 \leq j \leq n-1, \\
{\left[x_{0}, w\right]=\left[y_{i}, a_{i}\right] } & =c_{1}, \quad 1 \leq i \leq m, \text { and } \\
{\left[x_{0}, y_{i}\right] } & =c_{i}, \quad 2 \leq i \leq m .
\end{aligned}
$$

Now let $A=\mathfrak{f}_{n} \oplus\left\langle a_{i} \mid 1 \leq i \leq m\right\rangle, B=\mathfrak{h}_{m} \oplus\left\langle b_{i} \mid 1 \leq i \leq n-1\right\rangle, C=\left\langle c_{i} \mid 0 \leq i \leq m\right\rangle$ and $L=A \oplus B \oplus C$, with the products above. Note that $L$ is a nilpotent algebra of nilpotency $n, A$ and $B$ are nonabelian subalgebras of $L$, and $Z(L)=C$, so $\operatorname{dim} Z(L)=m+1$. Moreover, the derived algebra $[L, L]$ is nonabelian, and hence $L$ is not of the form treated in [5], but satisfies the hypotheses of our theorem.

\section{Application to free two-step algebras}

Suppose $F_{m}=\mathbb{C}^{m} \oplus \mathbb{C}^{\left(\begin{array}{c}m \\ 2\end{array}\right)}$ is the free two-step algebra on $m$ generators where $Z\left(F_{m}\right)=\mathbb{C}^{\left(\begin{array}{c}m \\ 2\end{array}\right)}$. It is not difficult to show that for $m>2$, the cohomology of $H^{*}\left(F_{m}\right)$ does not contain any free $\Lambda Z$ summands. However, using the theorem, we can see that $H^{*}\left(F_{m}\right)$ does contain many free $\Lambda C$ summands, for certain $C \subset Z$, as follows. Write $m=k+l$ for $k, l$ positive integers, and decompose $F_{m}$ as a direct sum of subalgebras as follows:

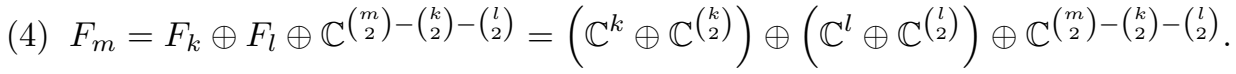

Here, the last summand is the repository for brackets of elements in $\mathbb{C}^{k}$ and $\mathbb{C}^{l}$. The decomposition (4) allows an application of the theorem with $A=F_{k}, B=F_{l}$

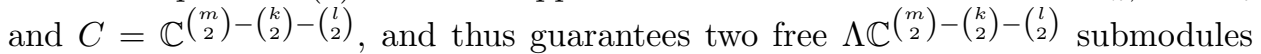
in $H^{*}\left(F^{m}\right)$. Combining these for different $k, l$ (valid when the co-generators are independent) actually yields the TRC for the algebras $F_{m}$ for $m \leq 5$, and gives an explicit method of constructing nontrivial cohomology classes in $H^{*}\left(F^{m}\right)$.

It is interesting to note that for $m \leq 5$, computer calculations show that the theorem predicts the maximal dimension of central subspaces $C$ for which there are free $\Lambda C$ summands in $H^{*}\left(F_{m}\right)$. We conjecture that this will hold for all $m$.

\section{REFERENCES}

1. Grant Cairns and Barry Jessup, New bounds on the Betti numbers of nilpotent Lie algebras, Comm. Algebra 25 (1997), no. 2, 415-430. MR.1428787 (98a:17032)

2. - Cohomology operations for Lie algebras, Trans. Amer. Math. Soc. 356 (2004), no. 4, 1569-1583 (electronic). MR2034319 (2005a:17018)

3. Ch. Deninger and W. Singhof, On the cohomology of nilpotent Lie algebras, Bull. Soc. Math. France 116 (1988), no. 1, 3-14. MR.946276 (90c:17023)

4. Stephen Halperin, Le complexe de Koszul en algèbre et topologie, Ann. Inst. Fourier (Grenoble) 37 (1987), no. 4, 77-97. MR927392 (89d:55040)

5. Hannes Pouseele and Paulo Tirao, Constructing Lie algebra homology classes, J. Algebra 292 (2005), no. 2, 585-591. MR2172169 (2006h:17028) 
6. Paulo Tirao, A refinement of the toral rank conjecture for 2-step nilpotent Lie algebras, Proc. Amer. Math. Soc. 128 (2000), no. 10, 2875-2878. MR1664387(2000m:17026)

7. - On the homology of graded Lie algebras, J. Pure Appl. Algebra 156 (2001), no. 2-3, 357-366. MR:1808831 (2001m:17026)

Department of Mathematics, La Trobe University, Melbourne, Australia 3086

E-mail address: G.Cairns@latrobe.edu.au

Department of Mathematics and Statistics, University of Ottawa, Ottawa, Canada K1N6N5

E-mail address: bjessup@uottawa.ca 